\title{
Gastric telangiectasis: a rare cause of severe blood loss in CREST syndrome
}

\author{
M.M. El-Omar, A.P. Jenkins, K. Hollowood, A.K. Banerjee and R.P.H. \\ Thompson
}

Gastrointestinal Laboratory, The Rayne Institute, St Thomas' Hospital, London SE1 7EH, UK

\begin{abstract}
Summary: A 46 year old woman presented with the CREST variety of systemic sclerosis and occult gastrointestinal bleeding due to vascular malformations of her stomach. Partial gastrectomy cured her anaemia. In systemic sclerosis, visceral angiography should be performed early when initial investigations have been negative.
\end{abstract}

\section{Introduction}

The CREST syndrome is a limited form of systemic sclerosis comprising calcinosis cutis, Raynaud's phenomenon, oesophageal fibrosis, sclerodactyly and telangiectases. The telangiectases are most commonly seen in the nail folds, palms and face but occasionally the gastrointestinal tract. ${ }^{1-3}$ In general, those associated with the CREST syndrome do not bleed, in contrast to those found in hereditary haemorrhagic telangiectasia. ${ }^{1,2,4}$ There have been five case reports of severe blood loss from upper gastrointestinal telangiectases in patients with scleroderma, of whom four had the CREST syndrome. ${ }^{3,5,6,7}$ We describe a patient in whom the diagnosis was only reached after visceral angiography.

\section{Case report}

A 46 year old woman presented in 1985 with iron deficiency anaemia, hypertension and Raynaud's phenomenon. Her haemoglobin was $11.7 \mathrm{~g} / \mathrm{dl}$ (normal 12-15) with a mean corpuscular volume (MCV) of $74 \mathrm{fl}$ (normal 80-100). She was started on nifedipine and ferrous sulphate. She represented in 1988 with severe Raynaud's phenomenon, digital tip ulceration, pain and swelling of her fingers and dyspepsia. Examination revealed thickening of the digital skin, telangiectases, and resorption and calcinosis of the terminal phalanges. The haemoglobin was now $7.6 \mathrm{~g} / \mathrm{dl}$ with an MCV of $61 \mathrm{fl}$. Gastroscopy revealed severe erosive gastritis confined to the antrum. A barium $\mathrm{X}$-ray showed mild gastro-oesophageal reflux,

Correspondence: M.M. El-Omar, B.Sc., M.R.C.P. Accepted: 19 August 1993 whilst sigmoidoscopy and colonoscopy were normal. Her serum was positive for anticentromere antibody.

A diagnosis of CREST syndrome was made and the patient was treated with ferrous sulphate and cimetidine, and prescribed heated gloves. She continued to suffer tiredness and painful swelling of her fingers, and was commenced on prednisolone $7.5 \mathrm{mg}$ per day with good effect. The serum ferritin levels remained low despite oral, and later intramuscular, iron. In December 1990, she developed a further episode of anaemia (haemoglobin $6.3 \mathrm{~g} / \mathrm{dl}$ ) requiring blood transfusion. A technetium-labelled red cell scan was negative and another colonoscopy and a small bowel meal were normal. A repeat gastroscopy in October 1991 again revealed florid haemorrhagic antral gastritis, although antral and duodenal biopsies were reported as normal. There was no evidence of Helicobacter pylori infection. When parenteral iron was stopped, severe anaemia recurred. A third gastroscopy in January 1992 revealed no change in the appearance of the antrum, and antral and duodenal biopsies were normal. Angiography of the coeliac and mesenteric arteries showed an area of abnormal vasculature in the inferior part of the greater curve of the stomach with dense staining of the stomach wall (Figure 1), suggesting a vascular malformation. In view of previous reports, ${ }^{8,9}$ prednisolone $20 \mathrm{mg}$ per day was given but without success.

In September 1992, the patient underwent a Billroth I gastrectomy. Histological examination of the resected specimen revealed focal areas within the antrum containing many thin-walled telangiectatic vessels within the lamina propria, a few of which were occluded by thrombus (Figure 2). There was some oedema, fibrosis and smooth muscle hypertrophy within the lamina propria, with a few scattered chronic inflammatory cells. 


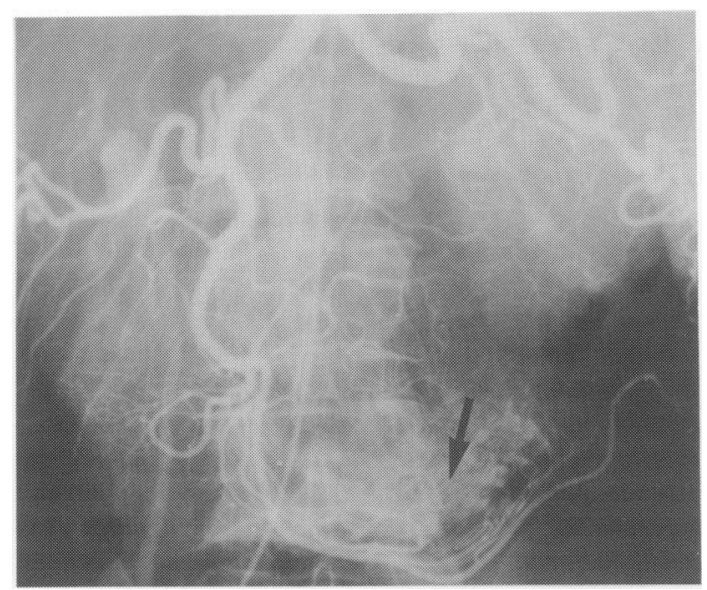

Figure 1 Coeliac axis arteriogram showing a vascular malformation arising from the right gastroepiploic artery (arrow).

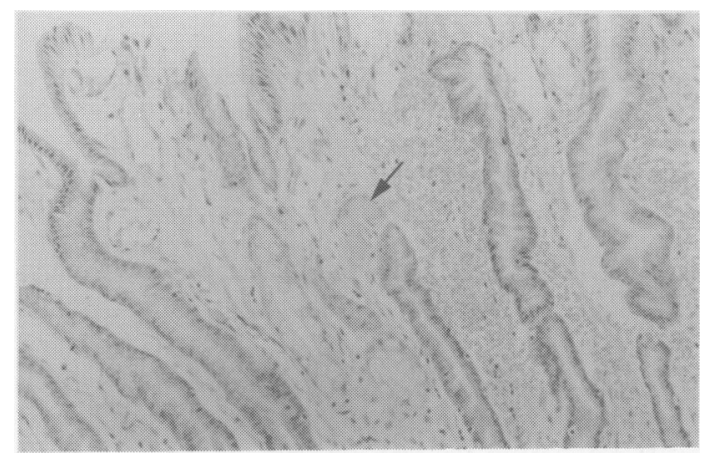

Figure 2 Antral mucosa showing numerous ectatic vessels. One vessel is occluded by thrombus (arrow).

The submucosa contained a few tortuous dilated veins. Review of previous antral biopsies confirmed the normal histology of those taken in January 1992, but those obtained in 1991 did show a few abnormally dilated capillary-type vessels in the lamina propria, one of which contained thrombus. The patient made an uneventful recovery and has since maintained a haemoglobin above $12 \mathrm{~g} / \mathrm{dl}$ without iron therapy.

\section{Discussion}

Telangiectasis of the gastrointestinal tract is known by a variety of terms, including vascular malformation, angiodysplasia, arteriovenous malformation, angioma and vascular ectasia. ${ }^{10}$ It is relatively uncommon in the upper gastrointestinal tract accounting for $2-5 \%$ of acute bleeding. ${ }^{10}$ In the CREST variety of systemic sclerosis, telangiectases occasionally occur in the gastrointestinal tract but rarely bleed. ${ }^{1,4}$ This is in contrast to hereditary haemorrhagic telangiectasia where the lesions often bleed profusely despite being histologically and endoscopically identical to those in CREST syndrome. ${ }^{4,10}$

In all four other reported cases of patients with CREST syndrome and severe gastrointestinal bleeding, ${ }^{3,5-7}$ gastric telangiectases were seen on gastroscopy, and in one the lesions were also demonstrated on angiography. ${ }^{5}$ In the present case, the diagnosis of gastric vascular malformation was only made by angiography. It is probable that the florid haemorrhagic antral gastritis seen in this case represented the vascular malformations. It is well known that histological recognition of such lesions in endoscopic biopsies is difficult. In retrospect, the normal biopsies taken in 1992 presumably reflect the focal nature of the ectatic vessels demonstrated in the resection specimen.

The endoscopic appearance of gastric vascular malformations varies. They are usually flat and well circumscribed with a surrounding halo, $2-7 \mathrm{~mm}$ in size, and may be single or multiple, occurring most commonly in the antrum. Occasionally they are more extensive and resemble the lines on a watermelon giving rise to the so-called watermelon stomach.' Diagnosis is usually made by upper gastrointestinal endoscopy, but the appearances were not diagnostic in our patient. Corticosteroids have been tried ${ }^{8,9}$ but were ineffective in our patient. They may achieve their beneficial effects by increasing the serum gastrin level, with its protective effects on the antral mucosa and the underlying abnormal vessels. ${ }^{8}$

Endoscopic coagulation of the lesions can be performed by laser or heater probe, but is only effective when there are a few lesions and may carry a small risk of bleeding, perforation or peritonitis. ${ }^{10}$ In conclusion, suspicion of a vascular malformation in the gut in patients with CREST syndrome is raised when initial investigations fail to reveal the source of bleeding. Visceral angiography is valuable and should be obtained early in those patients as gastroscopy may not be diagnostic. 


\section{References}

1. Seibold, J.R., Scleroderma, In: Kelley, W.N., Harris, E.D., Ruddy, S. \& Sledge, C.B. (eds) Textbook of Rheumatology. W.B. Saunders, Philadelphia, 1989, pp. 1215-1244.

2. Carr, R.D., Heisal, E.B. \& Stevenson, T.D. CRST syndrome: a benign variant of scleroderma. Arch Derm 1965, 92: 519.

3. Binford, R. CRST syndrome with gastrointestinal bleeding. Arch Derm 1968, 97: 603-604.

4. Winterbauer, R.H. Multiple telangiectasia, Raynaud's phenomenon, sclerodactyly and subcutaneous calcinosis: a syndrome mimicking hereditary haemorrhagic telangiectasia. Bull Johns Hopkins Hosp 1964, 114: 361-383.

5. Rosekrans, P.C.M., de Rooy, D.J., Bosman, F.T. et al. Gastrointestinal telangiectasia as a cause of severe blood loss in systemic sclerosis. Endoscopy 1980, 12: 200-204.
6. Marwick, T. \& Kerlin, P. Angiodysplasia of the upper gastrointestinal tract. J Clin Gastroenterol 1986, 8: 404-407.

7. Kolodny, M. \& Baker, W.G. CRST syndrome with persistent gastrointestinal bleeding. Gastrointest Endosc 1968, 15: 16-17.

8. Calam, J. \& Walker, R.J. Antral vascular lesion, achlorhydria and chronic gastrointestinal blood loss. Response to steroids. Dig Dis Sci 1980, 25: 236-239.

9. Jabbari, M., Cherry, R., Lough, J.O. et al. Gastric antral vascular ectasia: the watermelon stomach. Gastroenterology 1984, 87: 1165-1170.

10. Buchi, K.N. Vascular malformations of the gastrointestinal tract. Surg Clin North Am 1992, 72: 559-570. 